\title{
In silico Approach on Ribavirin Inhibitors for COVID-19 Main Protease
}

\author{
Adil Muala Dhumad 1(D), Hatem Jameel Majeed 2 (D), Kun Harismah 3, *D, Hasan Zandi ${ }^{\text {(D) }}$ \\ 1 Department of Chemistry, College of Education for Pure Sciences, University of Basrah, Basrah, Iraq; \\ adil.dhumad@uobasrah.edu.iq (A.M.D.); \\ 2 Pathological Analysis Technology Department, Al-Kunooze University College, Basrah, Iraq; hatam.j.m@kunoozu.edu.iq \\ (H.J.M.); \\ 3 Department of Chemical Engineering, Faculty of Engineering, Universitas Muhammadiyah Surakarta, Surakarta, \\ Indonesia; kun.harismah@ums.ac.id (K.H.); \\ 4 Department of Chemistry, Faculty of Science, University of Qom, Qom, Iran; hasan_zandi@yahoo.com (H.Z.); \\ * Correspondence: kun.harismah@ums.ac.id;
}

Scopus Author ID 56982926300

Received: 19.01.2021; Revised: 18.02.2021; Accepted: 23.02.2021; Published: 2.03.2021

\begin{abstract}
It has been well-known that COVID-19 has serious negative impacts on all sides of human life worldwide since the end of the year 2019, and exploring possible treatments for it is a must. To this aim, this computer-based in silico work was performed to investigate the impact of structural modification of Ribavirin (RBV) on its potential interaction with the COVID-19 main protease (MPO). Fourteen RBV compounds, including the original RBV and thirteen modified structures regarding the triazole ring, were investigated in this work based on quantum computations of structural chemistry features and molecular docking simulations (MDs) of RBV...MPO complex formations. Interestingly, six compounds were better than the original RBV for potent interaction with the MPO target, in which $\mathrm{R} 08\left(-\mathrm{NH}_{2}\right)$ was the best one among fourteen compounds. In conclusion, modification of RBV raised binding affinity against the COVID-19 MPO target.
\end{abstract}

Keywords: COVID-19; main protease; Ribavirin; inhibitor; docking; in silico.

(C) 2021 by the authors. This article is an open-access article distributed under the terms and conditions of the Creative Commons Attribution (CC BY) license (https://creativecommons.org/licenses/by/4.0/).

\section{Introduction}

Chemistry is almost the main resource of food and drug development and provides several other regular life needs. Among these, "structural chemistry" is one of the specified branches for examining chemical compounds' structural features to predict or to evaluate novel applications for different purposes [1]. For drug design and discovery purposes, structural chemistry could help very much by careful development of chemical compounds for pharmacotherapy treatments. Using computer-based methods could provide in silico environment to achieve the purpose [2]. In emergency cases, in silico structural chemistrybased protocols could help scientists evaluate the compounds for the targets in shorter times, avoiding the complications of experiments [3]. The procedure might also be applicable for exploring a possible treatment for the mysterious wide-spreading new corona-virus-disease (COVID-19), in which the world has been confused about how to deal with it since the late days of 2019 up to now [4]. In addition to prescribing self-care methods to prevent this pandemic disease, considerable efforts have been dedicated toexploring the pharmaceutical treatments for COVID-19 [5-8]. However, further investigations are still required for the process of drug discovery for COVID-19 [9-13]. Examining the potency of available drugs 
regarding the structural chemistry features for the pharmacotherapy of this mysterious disease is one way to find the lead compound among the already known hit compounds for such a purpose [14]. In the case of COVID-19, already known antiviral agents against HIV and HCV proteases have become as possible hit compounds to be examined against COVID-19 main protease (MPO) [15-18]. Indeed, characterizing the crystalline structure of MPO in early 2020 helped researchers to focus on this topic; the efforts have not been finished yet. The pandemic COVID-19 has made an emergency situation worldwide, and innovating an efficient pharmacotherapy protocol is necessary [19].

By examining the available compounds, structural chemistry features of Ribavirin (RBV) and its interaction with COVID-19 MPO have been investigated in this in silico work. RBV (1, $\beta$ D-ribofuranosyl-1,2,4-triazole-3-carboxamide) (Table 1) has already shown a variety of therapeutic activity against diseases caused by DNA and RNA viruses such as HCV [20]. RBV has also been used to treat respiratory syncytial virus infections, influenza A and B viruses, and Lassa fever virus [21]. Currently, RBV itself or in combination with other counterparts could work in a broad spectrum of antiviral agents with acceptable success [22]. Such advantages have encouraged researchers to examine the function of RBV for the treatment of COVID-19 [23]. The triazole part of RBV structure could be modified based on the structural chemistry concepts by substituting the current carboxamide group as a proposed site for a single-standing triazole structure [24]. In this case, such chemical modification could be done to evaluate the structural features of available RBV for possible potent interaction COVID-19 MPO target. To this aim, the formation and properties of a set of thirteen modified structures of RBV in addition to the original one have been investigated in this work by computer-based structural chemistry methods. Furthermore, functions of small RBV molecules against COVID-19 have been investigated through interactions with macromolecule MPO targets (Table 1 and 2, Figures 1 and 2). Hence, optimization of RBV for interacting with COVID-19 MPO target was investigated in this in silico work by the advantages of employing structural chemistry-based computational methods [25-30].

\section{Materials and Methods}

This work has been done to evaluate structural chemistry features of RBV to interact with COVID-19 MPO target based on the computer-based in silico approach. To this aim, the original RBV (R01) and a set of thirteen modified structures (R02-R14), following earlier work on the single-standing triazole [24], have been considered in this work (Table 1). As explained in Table 1, the current carboxamide group of traizole part of RBV has been substituted by other groups to generate new modified derivatives. Geometries of all fourteen RBV structures have been first optimized using the standard B3LYP/6-31G* level of density functional theory [31, 32] as implemented in the Gaussian program [33]. By these processes, stabilized structures with minimum energy have been obtained for further investigations. Moreover, single-point calculations have been performed using the B3LYP/6-31+G* level of theory to evaluate quantum descriptors for all stabilized structures. By these processes, properties such as molar volume (MV), energies of the highest occupied and the lowest unoccupied molecular orbitals (HOMO and LUMO), energy gap (EG), chemical potential $(\mu)$, hardness $(\eta)$, softness (S), and electrophilicity $(\omega)$ have been evaluated (Table 1$)$. Performing additional time-dependent and natural bond orbital calculations helped to evaluate HOMO and LUMO distribution patterns and molecular electrostatic potential (MEP) surfaces for the stabilized structures (Figure 1). Subsequently, 3D structure of COVID-19 MPO (ID: 6XBG) has been obtained from the 
Protein Data Bank [34], and it has been prepared for the molecular docking simulation (MDs) process using the SwissDock web server [35]. By performing blind MDs processes, the results, including delta- $\mathrm{G}$ of binding $(\Delta \mathrm{G})$, root-mean-square (RMS) deviation, and interacting amino acids (AA) have been evaluated for the interacting complexes of each of RBV compounds with the MPO target (Table 2 and Figure 2). By the advantage of employed in a silico environment, all of the required information for discussing the optimization of RBV for interacting with the COVID-19 MPO target has been obtained in this work.

Table 1. Ribavirin molecular features.

\begin{tabular}{|c|c|c|c|c|c|c|c|c|c|c|}
\hline Model & $\mathbf{R}$ & $M V$ & $D M$ & НОМО & LUMO & $E G$ & $\boldsymbol{\mu}$ & $\eta$ & $S$ & $\omega$ \\
\hline R01 & $-\mathrm{CONH}_{2}$ & 148.59 & 7.51 & -7.33 & -1.39 & 5.94 & -4.36 & 2.97 & 0.17 & 3.20 \\
\hline R02 & $-\mathrm{COOH}$ & 191.95 & 4.96 & -7.69 & -1.72 & 5.97 & -4.70 & 2.98 & 0.17 & 3.71 \\
\hline R03 & $-\mathrm{CHO}$ & 152.31 & 6.12 & -7.49 & -2.11 & 5.38 & -4.8 & 2.69 & 0.19 & 4.28 \\
\hline R04 & $-\mathrm{CH}_{3}$ & 169.21 & 3.18 & -7.31 & -0.41 & 6.90 & -3.86 & 3.45 & 0.14 & 2.16 \\
\hline R05 & $-\mathrm{CN}$ & 168.27 & 5.51 & -7.85 & -1.72 & 6.13 & -4.78 & 3.06 & 0.16 & 3.74 \\
\hline R06 & $-\mathrm{CFO}$ & 154.12 & 5.55 & -7.85 & -2.18 & 5.67 & -5.02 & 2.84 & 0.18 & 4.44 \\
\hline R07 & $-\mathrm{CF}_{3}$ & 149.97 & 4.34 & -7.76 & -1.12 & 6.64 & -4.44 & 3.32 & 0.15 & 2.97 \\
\hline R08 & $-\mathrm{NH}_{2}$ & 155.82 & 5.37 & -6.34 & -0.35 & 5.99 & -3.34 & 2.99 & 0.17 & 1.87 \\
\hline R09 & $-\mathrm{NO}_{2}$ & 154.87 & 5.69 & -7.91 & -2.98 & 4.93 & -5.44 & 2.46 & 0.20 & 6.01 \\
\hline R10 & $-\mathrm{OH}$ & 155.38 & 3.88 & -7.12 & -0.68 & 6.44 & -3.90 & 3.22 & 0.16 & 2.36 \\
\hline R11 & $-\mathrm{H}$ & 146.91 & 3.22 & -7.51 & -0.55 & 6.96 & -4.03 & 3.48 & 0.14 & 2.33 \\
\hline R12 & $-\mathrm{F}$ & 145.35 & 3.57 & -7.61 & -0.81 & 6.80 & -4.21 & 3.40 & 0.15 & 2.61 \\
\hline R13 & $-\mathrm{Cl}$ & 132.57 & 3.51 & -7.49 & -0.85 & 6.64 & -4.17 & 3.32 & 0.15 & 2.62 \\
\hline R14 & $-\mathrm{Br}$ & 146.11 & 3.43 & -7.35 & -0.86 & 6.49 & -4.10 & 3.24 & 0.15 & 2.60 \\
\hline
\end{tabular}

\section{Results and Discussion}

\subsection{Structural chemistry features.}

Within this work, fourteen RBV structures (R01-R14) have been investigated based on their traizole modification described in Table 1. Indeed, the carboxamide group of traizole, the original RBV (R01) was substituted with other functional groups following earlier work [24] to develop the modified structures. Such modification aimed to evaluate RBV for potent interaction with the COVID-19 MPO, which will be described in the following discussion. First, all the molecular structures were optimized to reach the minimum energy structure. Subsequently, their structural chemistry features were evaluated and examined (Table 1 and Figure 1). A quick look at the results of Table 1 could indicate that the RBV molecular features detected significant changes in structural modifications. The magnitudes of changes are more or less different for the modified compounds. The trend could show the importance of the effects of structural modifications on the characteristic features of molecular systems, which computer-based studies could very well recognize. R01 is the original RBV compound, and other modified compounds could be compared with this structure to evaluate the most potent RBV compound for the mentioned interaction purpose. Molar volume (MV) is an important parameter for the geometrical occupation of a molecule, and the results showed that such occupation could be changed for the modified structures by the reconfiguration of RBV according to the substituted group. The functional group size is important, in which sizes of 
atomic or molecular functional groups are different; however, other characteristics such as atomic electronegativity or molecular reconfiguration are also important for assigning the total volume feature. We tried to examine both groups to see the effects of such substitutions on the final behavior of interaction with the macromolecular target.

Among the structures, R02 $(-\mathrm{COOH})$ had the highest MV, and R13 $(-\mathrm{Cl})$ had the lowest MV. Besides volume, electric charge distribution at the molecular site is also an important parameter detected by dipole moment (DM). Different values of DM were obtained for the RBV compounds showing the effects of substituting functional groups on the molecular features, in which the effects were more or less significant regarding the original RBV structure (R01). Within the investigated RBV compounds, the maximum value of DM was obtained for $\mathrm{R} 01$, and the minimum value was obtained for $\mathrm{R} 10(-\mathrm{OH})$. The trend could show that structural analyses of chemical compounds are a multi-task work in which several parameters are required for such a purpose. To this point, examining values of the highest occupied and the lowest unoccupied molecular orbitals (HOMO and LUMO) and their corresponding parameters could approve such trend for multi-task analyses of chemical compounds. Each of HOMO and LUMO energy levels could work electron transfer parameters through ionization potential and electron affinity concepts. , The energy of the occupied level of HOMO, could determine the tendency of a molecule for electronic ionization, and energy of the unoccupied LUMO level could determine the tendency of a molecule for electronic affinity, which are both important parameters for assigning electronic reactivity for a molecular system. It was known that the modified structures should show different energy levels for HOMO and LUMO; however, the magnitudes of changes could be an important factor to assign the chemical features for each of RBV derivatives. By examining such values for all of fourteen compounds, R08 $\left(-\mathrm{NH}_{2}\right)$ was obtained to be in the best status regarding the level of HOMO and LUMO compared with other molecules. Energy values for HOMO and LUMO were almost in the steps of the best choice for energy conversion to make possible electron transferring for this molecule easier than other molecules and might increase its related reactivity. In addition to such quantitative analyses, the graphical representation for frontier molecular orbitals distribution patterns (Figure 1) also indicated that R08 had a typical shape, in which its HOMO and LUMO sites are apart from each other, both available for accepting/donoring electrons. Distribution patterns for other RBV compounds are similar to each other in graphical shapes but with a significant difference for R08. Furthermore, molecular electrostatic potential (MEP) surfaces also exhibited the charge distributions for the molecular systems ranging from the most negative to the most positive by Red, Yellow, Green, and Blue colors, respectively.

Besides the properties of pure HOMO and LUMO features, other properties such as energy gap (EG), chemical potential $(\mu)$, hardness $(\eta)$, softness $(S)$ and electrophilicity $(\omega)$ could be obtained to analyze the electronic properties of compounds following the structural chemistry features. EG is the energy difference between the HOMO and LUMO levels, in which its magnitude could help make judgments about the stability of a compound. $\mu$ is the tendency of the electronic system to escape from the equilibrium state. $\eta$ and $S$ indicate the difficulty and ease of electronic allocation for a compound, both related to reactivity. $\omega$ implies the tendency of a compound to receive electrons. By definitions of such parameters, it could be mentioned that each of HOMO and LUMO-related parameters could describe different aspects of the investigated chemical compounds. Indeed, frontier molecular orbitals could play important roles in the electronic structure recognition of chemical compounds. 


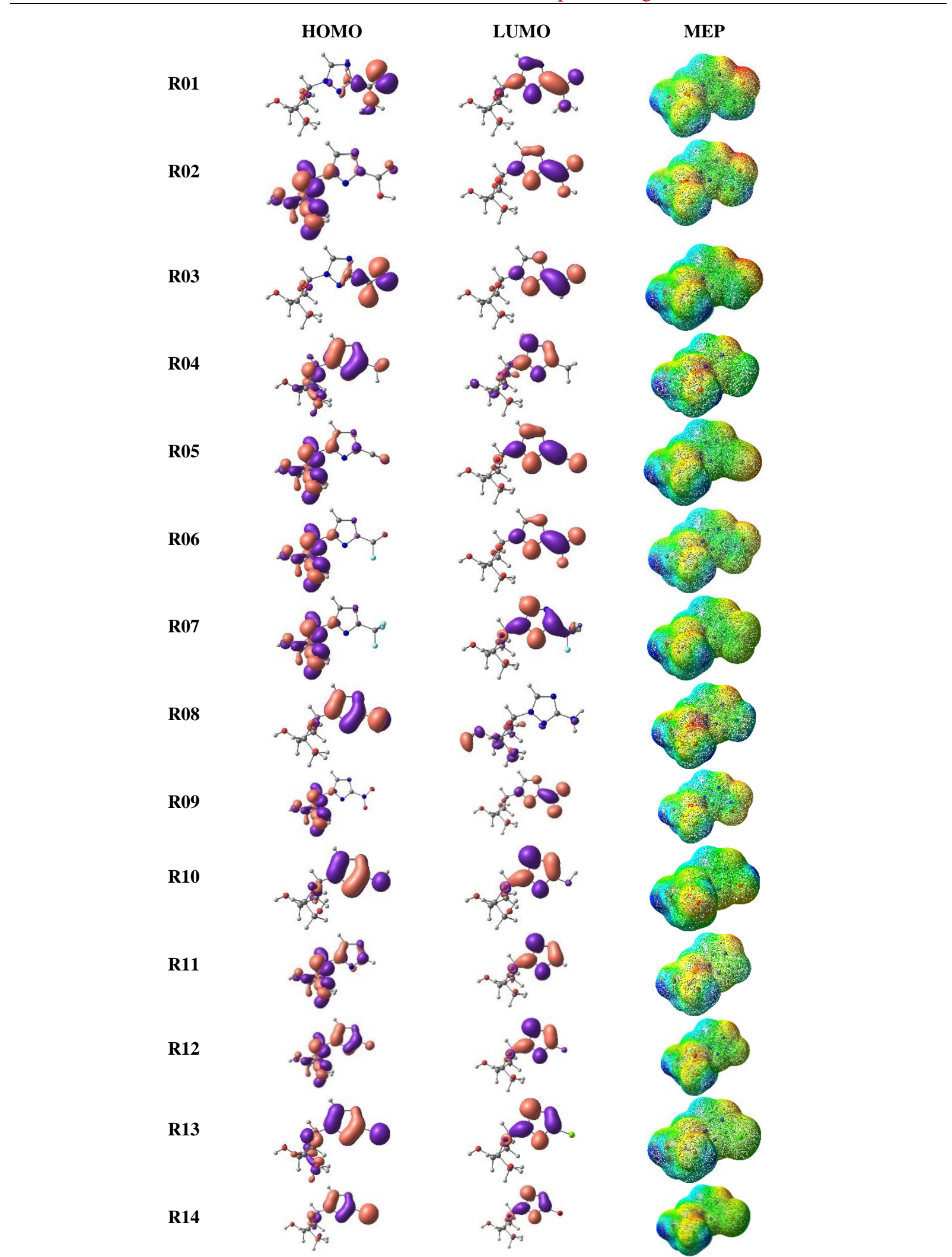

Figure 1. HOMO, LUMO, and MEP representations.

Specifically for R08, as the -supposed candidate of RBV compounds for interacting with the macromolecular target, the value $\omega$ of was very significant among the other compounds. Moreover, the values of $\eta$ and $S$ were also proper for such expected behavior. Values of EG and $\mu$ also supported the predictions. As concluding remarks of structural chemistry features. It could be mentioned that the molecular properties as very much important for characterizing the compounds for specific purposes. As discussed for fourteen RBV 
compounds, R08 was recommended as a distinguished compound for possible potent interaction with the macromolecular target. Indeed, such molecular features could provide potent ligands for the formation of ligand-target complex systems.

Table 2. Ribavirin models binding affinity.

\begin{tabular}{|c|c|c|c|c|}
\hline Model & $\Delta G \mathrm{kcal} / \mathrm{mol}$ & Rank & RMS & $\mathbf{A A}$ \\
\hline R01 & -6.30 & 7 & 24.12 & $\begin{array}{l}\text { HSD41, MET49, PHE140, LEU141, ASN142, GLY143, } \\
\text { SER144, CYS145, HSD163, HSE164, MET165, GLU166, } \\
\text { HSE172, ASP187, GLN189 }\end{array}$ \\
\hline R02 & -6.78 & 4 & 23.11 & $\begin{array}{l}\text { HSD41, MET49, PHE140, LEU141, ASN142, GLY143, } \\
\text { SER144, CYS145, HSD163, HSE164, MET165, GLU166, } \\
\text { HSE172, GLN189 }\end{array}$ \\
\hline $\mathbf{R 0 3}$ & -5.85 & 13 & 23.34 & $\begin{array}{l}\text { PHE140, LEU141, ASN142, GLY143, SER144, CYS145, } \\
\text { HSD163, HSE164, MET165, GLU166, HSE172, GLN189 }\end{array}$ \\
\hline R04 & -6.02 & 10 & 25.29 & $\begin{array}{l}\text { THR25, HSD41, SER46, MET49, ASN142, GLY143, } \\
\text { CYS145, HSE164, MET165, GLU166, GLN189 }\end{array}$ \\
\hline R05 & -6.06 & 9 & 22.13 & $\begin{array}{l}\text { PHE140, LEU141, ASN142, GLY143, SER144, CYS145, } \\
\text { HSD163, HSE164, MET165, GLU166, HSE172, GLN189 }\end{array}$ \\
\hline R06 & -6.91 & 3 & 24.67 & $\begin{array}{l}\text { GLU14, GLY15, MET17, VAL18, TRP31, ALA70, } \\
\text { GLY71, ASN95, LYS97, GLY120, SER121, PRO122 }\end{array}$ \\
\hline R07 & -5.99 & 12 & 26.56 & $\begin{array}{l}\text { THR24, THR25, TGR26, LEU27, HSD41, SER46, MET49, } \\
\text { ASN142, GLY143, CYS145 }\end{array}$ \\
\hline $\mathbf{R 0 8}$ & -7.89 & 1 & 28.98 & $\begin{array}{l}\text { GLU14, GLY15, CYS16, MET17, VAL18, TRP31, } \\
\text { ALA70, GLY71, LYS97, GLY120, SER121, PRO122 }\end{array}$ \\
\hline R09 & -6.69 & 5 & 25.12 & $\begin{array}{l}\text { HSD41, MET49, PHE140, LEU141, ASN142, GLY143, } \\
\text { SER144, CYS145, HSD163, HSE164, MET165, GLU166, } \\
\text { HSE172, GLN189 }\end{array}$ \\
\hline R10 & -5.99 & 11 & 25.44 & $\begin{array}{l}\text { THR25, THR26, LEU27, HSD41, MET49, ASN142, } \\
\text { GLY143, CYS145, GLN189 }\end{array}$ \\
\hline R11 & -6.48 & 6 & 33.81 & $\begin{array}{l}\text { ULE152, ASP153, TYR154, PHE294, VAL297, ARG298, } \\
\text { VAL303, PHE305 }\end{array}$ \\
\hline R12 & -5.71 & 14 & 26.45 & $\begin{array}{l}\text { THR24, THR25, THR26, LEU27, HSD41, MET49, } \\
\text { ASN142, GLY143, CYS145, GLN189 }\end{array}$ \\
\hline R13 & -7.06 & 2 & 29.21 & $\begin{array}{l}\text { GLU14, MET17, VAL18, TRP31, GLY15, ALA70, } \\
\text { GLY71, LYS97 }\end{array}$ \\
\hline R14 & -6.11 & 8 & 24.59 & $\begin{array}{l}\text { THR24, THR25, THR26, TYR118, ASN119, ASN142, } \\
\text { GLY143 }\end{array}$ \\
\hline
\end{tabular}

${ }^{*}$ Interacting complexes are represented in Figure 2.

\subsection{RBV ...MPO interacting complexes.}

To this point, no real therapeutic protocol has been found for the treatment of pandemic COVID-19, and it still has serious negative impacts on all parts of human life all around the world. Therefore, systematic investigations must innovate an efficient methodology for this mysterious disaster to human health quality. Earlier works proposed that RBV might be considered for the case of COVID-19 treatment [23]. To this aim, fourteen RBV derivatives (R01-R14) structures were investigated in this work to explore RBV...MPO interacting complex formations (Figure 2). Quantities and qualities of binding affinity of each of ligands against the macromolecular target were investigated using molecular docking simulation (MDs) processes to evaluate delta-G of binding $(\Delta \mathrm{G})$ and root-mean-square (RMS) in addition to interacting amino acids (AA) (Table 2 and Figure 2). The values of $\Delta \mathrm{G}$ were used to rank the complexes based on the most negative value for the best complex formation. RMS values were used to show the conformational variation of ligands to find the best position toward the target structure, in which a smaller value could refer to a smaller magnitude of variation for the conformational change of ligand. In addition, the quality of interactions could be followed by assigning interacting AA with the located ligand inside the RBV...MPO complexes. 


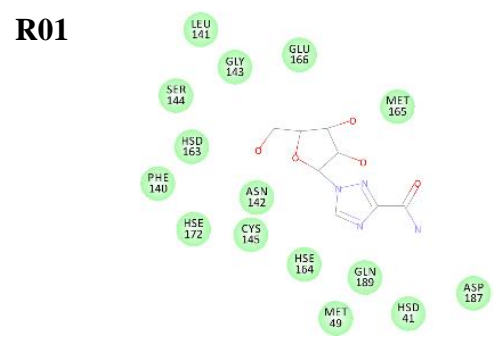

R02

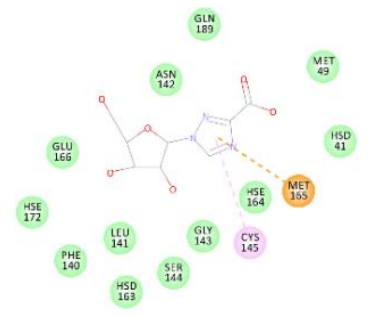

R03

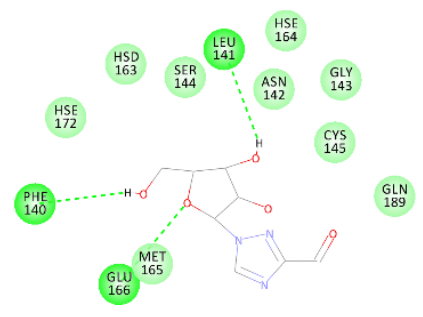

R04

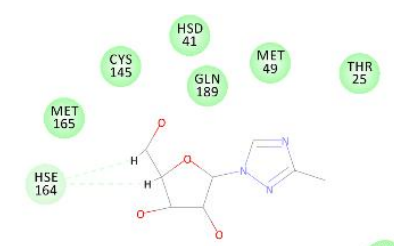

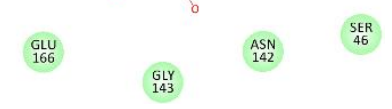

R05

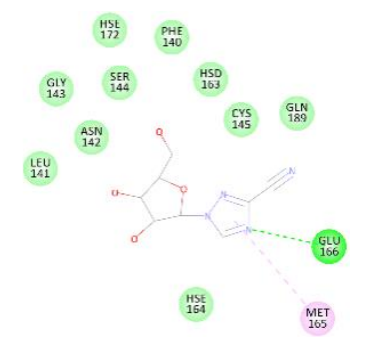

R06

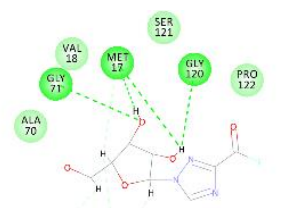

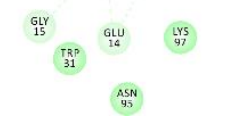

R07

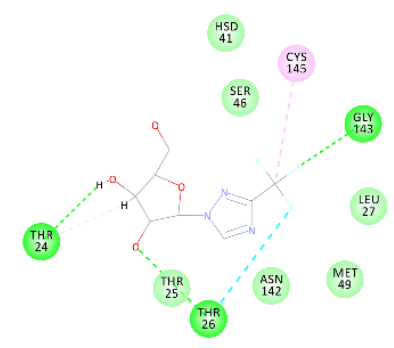

R08

$\left.\begin{array}{c}\text { CYS } \\ 16\end{array}\right)$

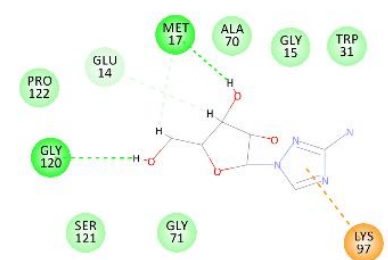

R09

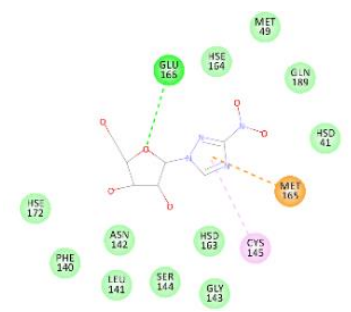

R10

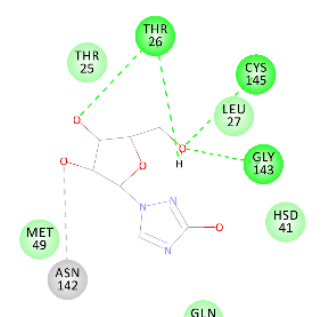

R11

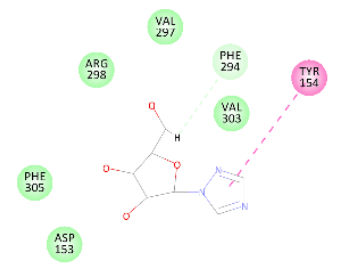

$(1452$

R12

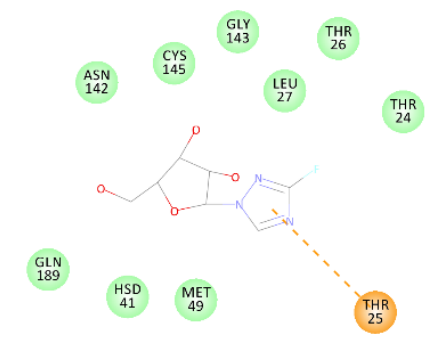

R13

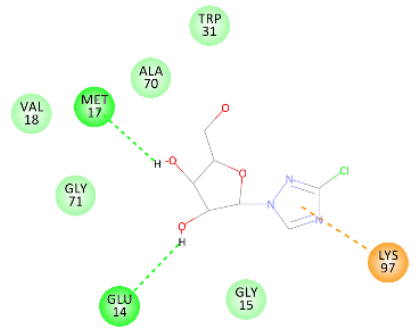

R14
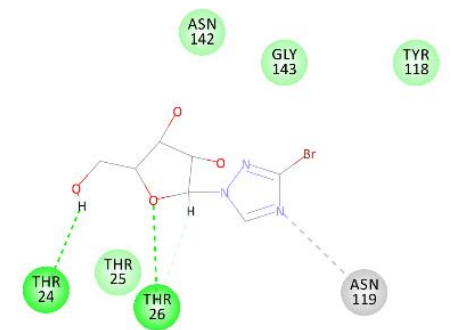

ASN
119

Figure 2. Interacting RBV...MPO complexes representations. 
It is worth noting that the mechanism of interactions could also be found by the results of the MDs processes.

The strength of interactions and types of interacting AA could be detected by the results at the molecular scale. A quick analysis of the results of Table 2 could show that the original RBV (R01) was not the most suitable one among the investigated ligands for potent interaction with the MPO target, placed at the Rank 7 of 14. Six of the other modified compounds could work better than R01 for contributing to potent interactions with the MPO target. R08 was placed at Rank 1 with the most suitable value of obtained $\Delta \mathrm{G}$ for RBV...MPO interacting complex systems. Remembering from the structural features, R08 was distinguished by the typical values of HOMO and LUMO and also their corresponding distribution patterns (Table 1 and Figure 1). The typical interacting features were also repeated for interacting R08...MPO complex formation. Other RBV compounds with a better tendency to interact with the target than the original compound were found: R13, R06, R02, R09, and R11 in the Ranks 2-6, respectively. The impact of modification of RBV on the interaction behavior was seen here by introducing R08 and five other ligands as better compounds for more potent interaction with the MPO target in comparison with the original RBV. Although $\Delta \mathrm{G}$ is an important factor for the strength of the complex formation, other parameters such as RMS are also important for the purpose. Examining RMS values for RBV compounds during the complex formation indicated that the structural modification also impacted conformational changes. The compounds showed different changes in this case. Sometimes, the functional groups of ligands could help the compound interact with different situations, which impacts structure on the corresponding function.

In the case of RBV compounds, those ligands with better values of $\Delta \mathrm{G}$ regarding the original RBV interacted with different sites of a macromolecular target, whereas those ligands with worse values of $\Delta \mathrm{G}$ regarding the original RBV interacted almost with more similar AAs. Interestingly, the lower values of RMS were obtained for worse ligands, but higher values of RMS were obtained for better ligands in comparison with the original RBV. To this point, the types and numbers of AAs were generally different for various interacting RBV...MPO complexes showing the importance of structural features on the activity of a compound. Such molecular scale results and graphical representations (Figure 2) could help make better judgments for the features and activity of the compound. As shown in Figure 2, R08 could interact with MPO through hydrogen bond and non-hydrogen bond interactions, in which the triazole ring also contributed to interactions in a very much better situation regarding the situation of original RBV (R01). Structural modification of RBV helped for the formation of complexes with more potent RBV...MPO interactions.

\section{Conclusions}

This computer-based in silico work was done to investigate the impact of RBV modifications on potent interaction with the COVID-19 MPO target. Based on the obtained structural chemistry features, R08 was distinguished regarding the values of HOMO and LUMO energy levels as dominant factors for ionization potential and electron affinity properties. Moreover, frontier molecular orbital distribution patterns proposed R08 as a typical ligand for complex formation with the MPO target. In addition to structural chemistry features, molecular docking results indicated that the original RBV was not so much suitable for complex formation with the target, whereas six modified compounds could work better than it for the purpose. Among which, R08 stood at the Rank 1 of RBV compounds for potent 
interaction with the MPO target to form the most stable interacting RBV...MPO complex system. Furthermore, RMS's values indicated that R08 had more conformational change than the original RBV for interaction with the target. Very much important to say that the interacting sites of MPO were different for R08 and the original RBV. Finally, structural modifications yielded $\mathrm{R} 08\left(-\mathrm{NH}_{2}\right)$ as a compound with more potent interaction with the MPO target than the original RBV, R01 (- $\left.\mathrm{CONH}_{2}\right)$.

\section{Funding}

This research received no external funding.

\section{Acknowledgments}

This research has no acknowledgment.

\section{Conflicts of Interest}

The authors declare no conflict of interest.

\section{References}

1. Randič, M.; Novič, M.; Plavšić, D. Solved and unsolved problems of structural chemistry. Boca Raton CRC Press; 2016.

2. Poroikov, V.V. Computer-aided drug design: from discovery of novel pharmaceutical agents to systems pharmacology. Biochemistry Supplement Series $B \quad$ 2020, $14, \quad 216-227$, https://doi.org/10.1134/S1990750820030117.

3. Schneider, G.; Clark, D.E. Automated de novo drug design: are we nearly there yet?. Angewandte Chemie International Edition 2019, 58, 10792-10803, https://doi.org/10.1002/anie.201814681.

4. Rothan, H.A.; Byrareddy, S.N. The epidemiology and pathogenesis of coronavirus disease (COVID-19) outbreak. Journal of Autoimmunity 2020, 109, https://doi.org/10.1016/j.jaut.2020.102433.

5. Qamar, M.T.; Alqahtani, S.M.; Alamri, M.A.; Chen, L.L. Structural basis of SARS-CoV-2 3CLpro and antiCOVID-19 drug discovery from medicinal plants. Journal of Pharmaceutical Analysis 2020, 10, 313-319, https://doi.org/10.1016/j.jpha.2020.03.009.

6. Harismah, K.; Mirzaei, M. COVID-19: A serious warning for emergency health innovation. Advanced Journal of Science and Engineering 2020, 1, 32-33, https://doi.org/10.22034/AJSE.2012032.

7. Wang, J. Fast identification of possible drug treatment of coronavirus disease-19 (COVID-19) through computational drug repurposing study. Journal of Chemical Information and Modeling 2020, 60, 3277-3286, https://doi.org/10.1021/acs.jcim.0c00179.

8. Shah, B.; Modi, P.; Sagar, S.R. In silico studies on therapeutic agents for COVID-19: Drug repurposing approach. Life Sciences 2020, 252, https://doi.org/10.1016/j.lfs.2020.117652.

9. Jiménez-Alberto, A.; Ribas-Aparici, R.M.; Aparicio-Ozores, G.; Castelán-Vega, J.A. Virtual screening of approved drugs as potential SARS-CoV-2 main protease inhibitors. Computational Biology and Chemistry 2020, 88, https://doi.org/10.1016/j.compbiolchem.2020.107325.

10. Harismah, K.; Mirzaei, M. Favipiravir: structural analysis and activity against COVID-19. Advanced Journal of Chemistry B 2020, 2, 55-60.

11. Mirzaei, M.; Harismah, K.; Da'im M.; Salarrezaei, E.; Roshandel, Z. Screening efficacy of available HIV protease inhibitors on COVID-19 protease. Journal of Military Medicine 2020, 22, 100-107, https://doi.org/10.30491/JMM.22.2.100.

12. Zhu, Y.; Li, J.; Pang, Z. Recent insights for the emerging COVID-19: drug discovery, therapeutic options and vaccine development. Asian Journal of Pharmaceutical Sciences 2020, in press, https://doi.org/10.1016/j.ajps.2020.06.001.

13. Senanayake, S.L. Drug repurposing strategies for COVID-19. Future Drug Discovery 2020, 2, https://doi.org/10.4155/fdd-2020-0010

14. Kandeel, M.; Al-Nazawi, M. Virtual screening and repurposing of FDA approved drugs against COVID-19 main protease. Life Sciences 2020, 251, https://doi.org/10.1016/j.lfs.2020.117627.

15. Jiang, H.; Zhou, Y.; Tang, W. Maintaining HIV care during the COVID-19 pandemic. The Lancet HIV 2020, 7, 308-309, https://doi.org/10.1016/S2352-3018(20)30105-3. 
16. Naghipour, S.; Ghodousi, M.; Rahsepar, S.; Elyasi, S. Repurposing of well-known medications as antivirals: hydroxychloroquine and chloroquine; from HIV-1 infection to COVID-19. 2020, 18, 1119-1133, https://doi.org/10.1080/14787210.2020.1792291.

17. Elfiky, A.A. Anti-HCV, nucleotide inhibitors, repurposing against COVID-19. Life sciences 2020, 248, https://doi.org/10.1016/j.lfs.2020.117477.

18. Havranek, B.; Islam, S.M. An in silico approach for identification of novel inhibitors as potential therapeutics targeting COVID-19 main protease. Journal of Biomolecular Structure and Dynamics 2020, in press, https://doi.org/10.1080/07391102.2020.1776158.

19. Ozkendir, O.M.; Askar, M.; Kocer, N.E. Influence of the epidemic COVID-19: An outlook on health, business and scientific studies. Lab-in-Silico 2020, 1, 26-30.

20. Nagaj, J.; Starosta, R.; Jeżowska-Bojczuk, M. Acid-base characterization, coordination properties towards copper (II) ions and DNA interaction studies of ribavirin, an antiviral drug. Journal of Inorganic Biochemistry 2015, 142, 68-74, https://doi.org/10.1016/j.jinorgbio.2014.09.019.

21. Loregian, A.; Scarpa, M.C.; Pagni, S.; Parisi, S.G.; Palù, G. Measurement of ribavirin and evaluation of its stability in human plasma by high-performance liquid chromatography with UV detection. Journal of Chromatography B 2007, 856, 358-364, https://doi.org/10.1016/j.jchromb.2007.05.039.

22. Brillanti, S.; Garson, J.; Foli, M.; Whitby, K.; Deaville, R.; Masci, C.; Miglioli, M.; Barbara, L. A pilot study of combination therapy with ribavirin plus interferon alfa for interferon alfa-resistant chronic hepatitis $\mathrm{C}$. Gastroenterology 1994, 107, 812-817, https://doi.org/10.1016/0016-5085(94)90131-7.

23. Hagar, M.; Ahmed, H.A.; Aljohani, G.; Alhaddad, O.A. Investigation of some antiviral N-heterocycles as COVID 19 drug: molecular docking and DFT calculations. International Journal of Molecular Sciences 2020, 21, https://doi.org/10.3390/ijms21113922.

24. Ozimiński, W.P.; Dobrowolski, J.C.; Mazurek, A.P. DFT studies on tautomerism of C5-substituted 1,2,4triazoles. Journal of Molecular Structure: THEOCHEM 2004, 680, 107-115, https://doi.org/10.1016/j.theochem.2004.05.005.

25. Mirzaei, M.; Elmi, F.; Hadipour, N.L. A systematic investigation of hydrogen-bonding effects on the $17 \mathrm{O}$, $14 \mathrm{~N}$, and $2 \mathrm{H}$ nuclear quadrupole resonance parameters of anhydrous and monohydrated cytosine crystalline structures: a density functional theory study. The Journal of Physical Chemistry B 2006, 110, 10991-10996, https://doi.org/10.1021/jp060301g.

26. Behzadi, H.; Hadipour, N.L.; Mirzaei, M. A density functional study of $17 \mathrm{O}, 14 \mathrm{~N}$ and $2 \mathrm{H}$ electric field gradient tensors in the real crystalline structure of $\alpha$-glycine. Biophysical Chemistry 2007, 125, 179-183, https://doi.org/10.1016/j.bpc.2006.07.010.

27. Samadi, Z.; Mirzaei, M.; Hadipour, N.L.; Khorami, S.A. Density functional calculations of oxygen, nitrogen and hydrogen electric field gradient and chemical shielding tensors to study hydrogen bonding properties of peptide group (OC-NH) in crystalline acetamide. Journal of Molecular Graphics and Modelling 2008, 26, 977-981, https://doi.org/10.1016/j.jmgm.2007.08.003.

28. Mirzaei, M.; Hadipour, N.L. Study of hydrogen bonds in 1-methyluracil by DFT calculations of oxygen, nitrogen, and hydrogen quadrupole coupling constants and isotropic chemical shifts. Chemical Physics Letters 2007, 438, 304-307, https://doi.org/10.1016/j.cplett.2007.03.011.

29. Tahmasebi, E.; Shakerzadeh, E. Potential application of B40 fullerene as an innovative anode material for Ca-ion batteries: in silico investigation. Lab-in-Silico 2020, 1, 16-20.

30. Khalid, H.; Hussain, R.; Hafeez, A. Virtual screening of piperidine based small molecules against COVID19. Lab-in-Silico 2020, 1, 50-55.

31. Zhang, I.Y.; Wu, J.; Xu, X. Extending the reliability and applicability of B3LYP. Chemical Communications. 2010, 46, 3057-3070, https://doi.org/10.1039/C000677G.

32. Ditchfield, R.H.; Hehre, W.J.; Pople, J.A. Self-consistent molecular-orbital methods. IX. An extended Gaussian-type basis for molecular-orbital studies of organic molecules. The Journal of Chemical Physics 1971, 54, 724-728, https://doi.org/10.1063/1.1674902.

33. Frisch, M.J.; Trucks, G.W.; Schlegel, H.B.; Scuseria, G.E.; Robb, M.A.; Cheeseman, J.R.; Scalmani, G.; Barone, V.; Mennucci, B.; Petersson, G.A. Gaussian 09 D.01 Program. Gaussian. Inc.: Wallingford, CT, USA. 2009.

34. Burley, S.K.; Berman, H.M.; Bhikadiya, C.; Bi, C.; Chen, L.; Di Costanzo, L.; Christie, C.; Dalenberg, K.; Duarte, J.M.; Dutta, S.; Feng, Z. RCSB Protein Data Bank: biological macromolecular structures enabling research and education in fundamental biology, biomedicine, biotechnology and energy. Nucleic Acids Research 2019, 47, 464-474, https://doi.org/10.1093/nar/gky1004.

35. Grosdidier, A.; Zoete, V.; Michielin, O. SwissDock, a protein-small molecule docking web service based on EADock DSS. Nucleic Acids Research 2011, 39, 270-277, https://doi.org/10.1093/nar/gkr366. 